ary experiments have been conducted on the effect of speeding-up the ordinary eard with the ultimate object of obtaining information that will enable the industry, with its 20,000 cards to exploit most economically and effectively the modern trends.

In the Sizing Room could be seen the results of some experiments on a new method of fibre laying on sized warps. This is an extension of work on the selfbrushing of warps, which has also been continued to the point where the latest version of the attachment is available commercially.

In the Weaving Shed the latest modifications to the Shirley loom were demonstrated : it has now been fitted with a re-designed positive dobby using a paper pattern control in place of the earlier system using lags and pegs, and these modifications enable the dobby to operate at high speeds. Work on loom mechanisms, especially as they are affected by the demands for ligh speeds, has been carried out in collaboration with British Northrop Ltd. Visitors were shown a device for the hot-stretching of tyre cords which also shows considerable promise in other directions where the rapid application of dry heat at a uniform temperature is desirable : this is the fluidized bed. Present work is concentrated on the dyeing of fabrics from synthetic fibres such as nylon or 'Terylene' where the only major technical problem remaining is the application of the dye liquor in suitable form and suitable quantity. Arrangements have been made for the fluidized bed to be incorporated in a commercial frame for the heat treatment of bulked yarns at high speed.

The increasing shortage of water, its high cost, and the increasing difficulty and expense of disposing of effluent all make the efficient washing of textiles in finishing of great importance. It has been estimated that the cost of water may well be trebled in the next 5-10 years. A theoretical basis for the removal of water-soluble impurities has been established by Institute staff, together with some practical confirmation, and this will be extended as soon as possible to full-scale operation and extended to the removal of insoluble contamination. Much work remains to be done here to provide the most efficient and economical procedures, but good progress has been made.

Chlorite bleaching, which is becoming increasingly important, has been the subject of much work at the Institute in recent years. Conditions for its use under non-corrosive and non-poisonous conditions have been established, and the use of activators of different kinds has been investigated resulting in the development of a now method of activating chlorite which permits the use of a cold-pad method for chlorite bleaching. This is particularly valuable for blends including fibres sensitive to the more normal vigorous bleaching processes.

The programme of work on fabrics from blended fibres has now reached the stage where the Institute can expect to be able to forecast most of the properties of such fabries from a knowledge of the behaviour of the individual fibres. From now on, therefore, it will be necessary to work only on the individual properties of yarns and fabrics from 100 por cont single fibre form.

As part of a general investigation of the relation between the molecular constitution of fibres and their textile properties, a number of silk fibroins of different origins have been examined by chemical and X-ray methods. Fundamental investigations of the geometrical structure of continuous filament yarns and of the effect of twist on tensile properties have also been undertaken.

Fundamental work on stiffness, crease-rosistance and crease-recovery is being carried out at the Institute, and exhibits were concerned with the relations between fibre, yarn and eloth stiffness. the effect of crease orientation on creasability, the effect of time in the measurement of creasability, ageing after finishing, and the measurement of the forces involved in creasing and crease-recovery.

The foregoing are but some of the many aspects of the Institute's work which were on show. The attendance figures, coupled with the enthusiasm and interest of the visitors to the open days, contributed to their success and are both an encouragement to the research staff and proofs of the continuing esteem in which the Institute is held.

\title{
SOLAR FLARES
}

COLAR flares were described by Prof. M. A. Ellison $S$ in his Friday Evening Discourse at the Royal Institution on October 23 as catastrophic events in the hot, outer layers of the Sun. These have immediate repercussions in our terrestrial atmosphere 93 million miles away. A flare emits simultaneously both waves and particles; but the waves, irrespective of wave-length, travel with the speed of light and arrive together after an interval of $8 \frac{1}{2}$ min., whereas the particles lag behind, arriving after various intervals depending on their individual speeds. The particles consist of three main recognizable groups. The first, the magnetic storm particles, which reach our atmosphere after some $20-50 \mathrm{hr}$., consist mainly of ionized hydrogen atoms--protons - and electrons, and form a plasma. Of low energy and guided by tho Earth's magnetic field these particles enter our atmosphere in the regions close to the magnetic poles, setting up powerful electric currents in the ionosphere and hence magnetic storms. In addition, they excite the atoms and molecules in the atmosphere and produee tho colourful displays of the auroræ.
The second group of particles, consisting mainly of protons with energies of $10^{7}$ to $4 \times 10^{8} \mathrm{eV}$., take about $1 \mathrm{hr}$. or more to arrive, and are to be regardod as cosmic rays. They enter the atmosphere at locations within $30^{\circ}$ of the two geomagnetic poles and give rise to intense ionization in the $D$-region of the ionosphere, producing polar-cap black-outs of incoming cosmic radio noise in the very-high-frequency band. They do not possess, however, sufficient energy to penotrate to the lower levels of the atmosphere or to generate secondary particles and thus can be studied directly only from high-flying balloons or indirectly from the absorption effects of radio waves which their ionization produces in the polar regions.

On twelve occasions during tho past twenty yearsthe first on February 28, 1942-meson monitors (maxirnum response to particles of about $10^{10} \mathrm{eV}$.) and noutron monitors (maximum response to particles of about $10^{9} \mathrm{eV}$.) located at ground-level have recorded sudden increases of cosmic-ray intensity. The showors of primary particles responsible for the increases came directly from the Sun and all occurred 
shortly after the peak intensity of outstanding solar flares. The interval between the peak brightness and the arrival of the particles-high energy $\left(10^{9}\right.$ to $10^{11}$ eV.) cosmic-ray protons-at the Earth varied between $3 \mathrm{~min}$. and $\mathrm{I} \mathrm{hr}$. These high-energy particles constitute the third group and indicate that in solar flares there exists a mechanism by which particles can be accelerated to relativistic energies.

The enormous energy-about $10^{38}$ ergs-which is released during a few minutes by an intense solar flare is greater than the entire heat-energy stored up within the solar corona and chromosphere. It cannot, therefore, have been drawn in from the surrounding regions, but must have been stored up slowly in situ, almost certainly in the complex magnetic fields permeating the flare region above a large sunspot. It is now recognized that the magnetic lines of force in the flare region are anchored in a plasma of ionized particles, which is in a state of flux, but the mechanism by which the energy is slowly stored in the plasma and then explosively released is not fully understood. About 1 per cent of the energy released is used to accelerate the high-energy particles.

The positions of Class 3 and $3+$ flares, in general, are distributed uniformly with respect to the Sun's central meridian, but the positions of the intense cosmic-ray flares which have occurred during the past twenty years are such that seven are located on, or near, the west limb of the solar disk and none nearer than $75^{\circ}$ to the east limb. This longitudinal asymmetry and strong preponderance in the western hemisphere means that there is a greater probability that the cosmic rays accelerated into space by a flare west of the meridian will reach the Earth and produce ground-level effects. Since high-energy particles are guided to their destinations by magnetic fields, there must exist at these times a radial magnetic field in the Sun-Earth space and the lines of force along which the particles spiral in towards the Earth must be bent by the solar rotation convex to the west.

When the particles enter the Earth's magnetic field, their trajectories are bent into complicated Störmer orbits, depending on their energies and their directions of travel relative to the Earth's magnetic axis. The positions of the impact zones where the intensities are much greater than elsewhere have been observed and the directions of the original trajectories before entering the Earth's field have been computed for many of the showers. The showers have all been found to approach the Earth from a limited region of the sky containing the Sun.

The particles are accelerated during the flare flash, which is probably all over within a few minutes; but the cosmic rays in some cases continue to arrive at the Earth for as long as $24 \mathrm{hr}$. after the flare has died away. This is strong evidence for the trapping and storage of the particles in the interplanetary magnetic fields. These interplanetary fields also modify the 'constant' incoming flux of galactic cosmic rays. For example, at the commencement of a magnetic storm there is a sudden decrease of cosmic-ray intensitythe Forbush decrease-when the primary particle radiation reaching the Earth may be reduced by as much as 30 per cent and may not return to normal for ten days or more. The Earth is apparently trapped inside a 'magnetic bottle', formed by lines of magnetic force which are drawn out into space from a sunspot region by the issuing clouds of storm particles, and within this bottle the Earth is shielded from the normal cosmic-ray flux entering from the galaxy. Particles of lowest energy suffer the greatest reduction of intensity. Furthermore, if when a solar flare oceurs within the neck of the bottle, it is assumed that the bottle is bent by the solar rotation convex towards the west and that, at its point of anchorage in the Sun, the neck subtends a solid angle of about one radian, we have a model with which to explain in general terms the longitude asymmetry characteristic of the cosmic-ray producing flares.

The discovery that the Sun accelerates into space particles with energies as high as $10^{11} \mathrm{eV}$. suggests that the origin of cosmic rays is to be sought in specific astronomical bodies, such as the atmospheres of flare-active stars, in supernovæ explosions and in objects like the Crab nebula where powerful magnetic fields anchored in turbulent, unstable plasmas are known to exist. However, treating the Sun as an 'average star' in regard to its cosmic-ray output leads to too small a value by a factor of $10^{6}$ for the total injection of cosmic rays into galactic space from stellar sources. Nor can solar showers account for the acceleration of the very high-energy particles up to $10^{19} \mathrm{eV}$. These problems, together with the questions as to how the high-energy particles are contained within the galactic magnetic fields and whether the particles we receive are generated within our own galaxy or have crossed inter-galactic spaces, still require elucidation.

\section{FUNCTIONS OF PROTEIN MOLECULES}

$$
\mathrm{T}
$$
HE British Biophysical Society held a discussion on the "Functions of Protein Molecules" on December 13, 1961, as part of their Christmas meeting at the Royal Institution in London. Instead of presenting a diffuse discussion of all possible aspects of biological activities of proteins the symposium was concerned with a fairly detailed treatment of some selected subjects.

The session was introduced by Prof. A. Neuberger, who issued a timely warning about too circumscribed discussions of active sites of protein molecules. He quoted recent work from Prof. Rossi-Fanelli's laboratory which showed that though hæmoglobin still reacted rapidly and reversibly with oxygen when some amino-acid residues had been removed, impor. tant changes in the kinetics of the reaction occurred after such chemical modification. The first main speaker was Prof. Q. H. Gibson (Sheffield), who gave a most interesting survey of "Hæm-protein and Hæmoprotein-ligand Reactions". An impressive range of techniques has been applied to the study of the mechanisms of the reactions of globin with hæm and of hæmoglobin with oxygen, carbon monoxide and other ligands. Globin from human hæmoglobin is a heat-labile protein of molecular weight 33,000 which combines with hæm in a rapid and complex reaction via an intermediate complex:

2 globin $+4 \mathrm{hæm} \rightleftharpoons$ (intermediate complex $) \rightarrow$ hæmoglobin

to form the relatively stable hæmoglobin of molecular weight 68,000 . 